\title{
Geomóvel: Um Aplicativo para Auxílio a Aulas de Campo de Geologia
}

\author{
Edgar Marçal ${ }^{1}$, Rossana M. C. Andrade ${ }^{1}$, Windson Viana ${ }^{1}$, Daniel Rodrigues ${ }^{2}$, \\ Márcio Maia ${ }^{1}$, Wendell Mendes ${ }^{3}$, Jamile Freire ${ }^{2}$, Witalo Benicio ${ }^{4}$ \\ ${ }^{1}$ Mestrado e Doutorado em Ciência da Computação - Universidade Federal do Ceará \\ (UFC) - Fortaleza, CE - Brasil \\ ${ }^{2}$ Departamento de Geologia - Universidade Federal do Ceará (UFC) - Fortaleza, CE - \\ Brasil \\ ${ }^{3}$ Instituto Universidade Virtual - Universidade Federal do Ceará (UFC) - Fortaleza, CE \\ - Brasil \\ ${ }^{4}$ Campus da UFC em Quixadá - Universidade Federal do Ceará (UFC) - Quixadá, CE \\ - Brasil

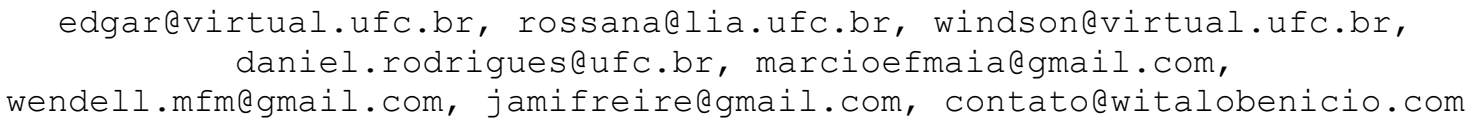

Abstract. Mobile devices has proven important tools to aid teaching and learning, to enable access to activities and content virtually anywhere and anytime. A pedagogical practice that can benefit from the features of these devices is the field class, common in many graduations in science. This paper presents the design and development of a mobile system that assists students in the undergraduate program during these field classes through the use of features such as compass, accelerometer, GPS and digital camera embedded in these devices. The goal of the system is to extend the benefits of the class field, equipping the student with a tool in which it can do so integrated multimedia annotations (text, image and sound). Subsequently, the student views all notes made and the course of the class through the Google Earth software. A case study was conducted with undergraduate students in geology and the initial results show advantages in the use of the application developed.

Resumo. Dispositivos móveis tem se comprovado ferramentas importantes para o auxílio ao ensino e à aprendizagem, ao permitir acesso a atividades e a conteúdo praticamente em qualquer lugar e a qualquer momento. Uma prática pedagógica que pode se beneficiar dos recursos desses dispositivos é a aula de campo, comum em muitas graduações em Ciências. Este trabalho apresenta a concepção e o desenvolvimento de um sistema móvel que auxilia os alunos do curso de graduação durante essas aulas de campo, através da utilização de recursos como bússola, acelerômetro, GPS e câmera digital embutidos nesses dispositivos. O objetivo do sistema é ampliar os benefícios da aula de campo, equipando o estudante com uma ferramenta na qual ele pode fazer de maneira integrada anotações multimídia (texto, imagem e som). Posteriormente, o estudante consulta todas as anotações feitas e o percurso da aula através do software Google Earth. Um estudo de caso foi realizado com alunos do curso de graduação em Geologia e os resultados iniciais demonstram vantagens no uso do aplicativo desenvolvido. 


\section{Introdução}

Em todo o Brasil, os índices de evasão no curso de Geologia são elevados de 50 a $70 \%$. Durante o primeiro ano do curso, as disciplinas de Geologia Geral representam o contato inicial com o tema central do curso e as primeiras aulas de campo que procuram simular atividades de coleta e observação comuns aos Geólogos. Aprimorar as práticas pedagógicas dessas disciplinas desperta uma curiosidade maior por parte dos alunos e os ajuda a surpassar as dificuldades de adaptação à universidade e de acompanhamento das disciplinas básicas presentes nos primeiros semestres ${ }^{1}$.

Uma potencial forma de tornar as aulas dessas disciplinas ainda mais instigantes e motivadoras é uso de tecnologias da informação. Em especial, destacam-se os sistemas de informação geográficos e as tecnologias de computação móvel e ubíqua. Estando essas últimas já presentes no cotidiano de muitos dos alunos que utilizam seus celulares e smartphones para diversas atividades de comunicação e entretenimento. A aprendizagem auxiliada por computação móvel (em inglês, m-learning) surge como uma tecnologia inovadora que utiliza dispositivos móveis (DMs), como smartphones e tablets, durante o processo de aprendizagem. Vários autores já comprovaram os benefícios do uso desses dispositivos para propósitos educacionais e demonstraram que a introdução da computação móvel na sala de aula motiva o aprendizado, a colaboração e a comunicação entre os alunos [Sharples, 2007; Viana et al., 2011; Sanchez et al., 2009; Marçal et al., 2010]. Na verdade, os DMs transformaram-se em plataformas importantes para auxiliar a aprendizagem, devido à sua onipresença, ao considerável poder de armazenamento e processamento, aos seus recursos multimídia (e.g., áudio e vídeo) e de seus sensores (e.g., acelerômetro, GPS e bússola). Além disso, as diversas formas de comunicação (e.g., 3G, Wi-Fi) possibilitam aos alunos o acesso a conteúdos educacionais independentemente de local e horário pré-estabelecidos.

Dentro desse contexto, este trabalho apresenta um sistema para DMs que visa apoiar aulas de campo de Geologia: o Geomóvel. Este aplicativo pretende integrar de maneira simplificada, organizada e lógica as informações coletadas durante as aulas de campo. Desta forma, busca-se desenvolver uma ferramenta que auxilie a aprendizagem do aluno ao facilitar o registro das informações em campo, diminuindo a duração desta tarefa e, consequentemente, proporcionando a ele mais tempo para dedicar-se aos aspectos diretamente relacionados ao estudo da Geologia.

O restante deste artigo é dividido como segue: a Seção 2 apresenta a metodologia de desenvolvimento e o levantamento de requisitos do aplicativo Geomóvel. A Seção 3 detalha suas funcionalidades e o processo de exportação de dados do aplicativo. A Seção 4 apresenta um estudo exploratório de uso do Geomóvel realizado com alunos de Geologia. Por fim, a Seção 5 contem as considerações finais e os trabalhos futuros.

\section{Metodologia de Desenvolvimento}

Considerando os aspectos multidisciplinares envolvidos no desenvolvimento de uma ferramenta computacional voltada para favorecer o ensino de Geologia, optou-se por adotar a metodologia Co-Design proposta por Millard et al. (2009) - Figura 1. Esta

1 I e III Seminário Nacional sobre Cursos de Graduação em Geologia http://ppegeo.igc.usp.br/pdf/ted/v1n1/v1n1a11.pdf e http://www.ige.unicamp.br/terraedidatica/v1/pdfv1/p051-054_sobreira.pdf 
metodologia promove a participação dos especialistas no domínio (e.g., professores de Geologia) desde o início do Projeto. Quatro dos seus estágios focam na elicitação dos requisitos e consideram a constante troca de conhecimentos entre as equipes.

\subsection{Delimitação de Escopo e Troca de Conhecimento}

Nessas primeiras fases da metodologia, reuniões se estabelecem entre as equipes de especialistas para troca de conhecimento e delimitação do problema a ser abordado. Foram mostrados aos professores de Geologia, por exemplo, diversas aplicações de $m$ learning usando GPS e multimídia que teriam potencial para auxiliar as aulas. Decidiuse, então, em conjunto, abordar em um primeiro momento a automatização das atividades de coleta de dados das aulas de campo.

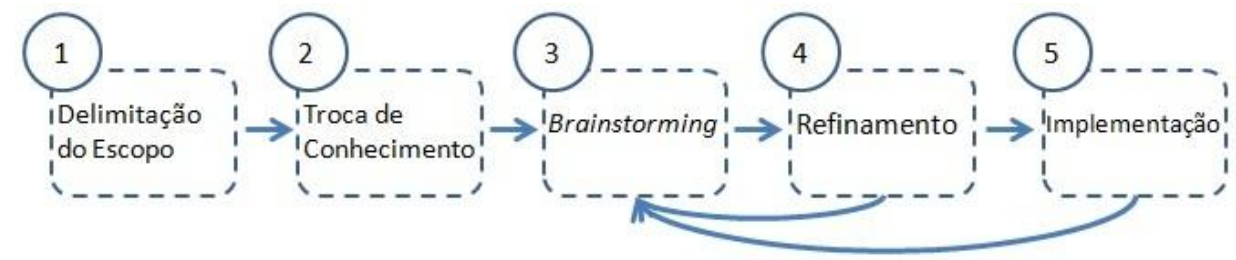

Figura 1- Etapas da Metodologia Co-Design adaptado de Millard et al. (2009).

Em Geologia, as aulas de campo são atividades práticas essenciais ao aprendizado. Estas aulas, em geral, requerem dos alunos a aquisição de dados descritivos e interpretativos relacionados ao contexto da distribuição das rochas, além de outros fatores associados: como sua influência na conformação do relevo, seu conteúdo mineral e paleontológico, suas condições portadoras de fluidos (águas subterrâneas e hidrocarbonetos), suas estruturas e sua evolução [Teixeira et al., 2000].Em muitos cursos, desde o primeiro semestre o aluno já tem contato com as aulas de campo (e.g., disciplina de Geologia Geral I). Ao longo do curso, várias outras disciplinas utilizam as aulas de campo, como: Paleontologia, Geologia Estrutural e Geotectônica.

As informações coletadas nas aulas de campo dizem respeito a objetivos específicos da matéria estudada (e.g., tipo de rocha, minerais, estruturas, fósseis), assim como, a descrição do itinerário (quilometragem do veículo, ponto de partida). Todas essas anotações são feitas em uma caderneta de campo antes de sua transferência para o relatório da viagem. Parte dessas informações é fornecida pelo professor e outra parte os próprios alunos devem coletar. Além destas anotações feitas em caderneta, incluem-se também aquisição de fotografias e de coordenadas geográficas (por GPS). As dificuldades mais citadas pelos alunos em tarefas de campo dizem respeito à organização das informações e sua padronização. Neste ambiente de estudo, que inclui cortes em beira de estradas, minas e outras regiões externas, outro fator complicador é a dificuldade imposta pelo meio em anotar as informações durante a aula. Isso ocorre também em função do próprio número de alunos, quando elevado, e da necessidade do uso de diferentes ferramentas (caderneta, câmera digital, bússola, GPS etc.) que não funcionam de forma integrada e apresentam alto custo financeiro.

\subsection{Brainstorming e Refinamento dos requisitos}

Após as primeiras reuniões entre as equipes de desenvolvimento de sistemas e de Geologia, decidiu-se realizar uma experiência exploratória de uso de tablets durante aulas de campo. O objetivo era observar como seria a utilização de tablets em uma aula 
de campo real, avaliando sua praticidade, facilidade de uso e os recursos do dispositivo. Para prática, um tablet foi configurado com recursos que poderiam ajudar na aula de campo: um aplicativo simulador de bússola, a câmera digital, uma aplicação para fazer anotações textuais e um aplicativo para capturar a localização pelo GPS. O tablet foi utilizado em uma expedição de 12 dias no interior do Ceará por um aluno monitor, de modo a não afetar as atividades dos alunos regulares. $\mathrm{O}$ monitor realizou algumas das atividades de campo comumente feitas pelos alunos: tirar fotos, anotar informações, medir atitudes (ângulos de fraturas) e coletar coordenadas geográficas.

As dificuldades encontradas durante esse teste foram importantes para a definição dos requisitos do Geomóvel e para elaboração da sua primeira versão. Os principais problemas relatados pelo monitor foram: i) os dados eram coletados por diferentes aplicativos e não era possível integrá-los ou relacioná-los; ii) algumas das aplicações utilizadas não registravam e nem exportavam os dados coletados, o que dificultava a sua visualização após a viagem; iii) em alguns momentos, devido à forma da rocha, não era possível realizar uma medição e ver os valores na tela do tablet devido ao seu tamanho ou ao ângulo exigido para a medição; iv) a luminosidade ambiente excessiva, em certos instantes, dificultava a entrada textual das informações e a precisão para a realização das fotografias; e, v) o tamanho do tablet utilizado (10"), atrapalhava seu manuseio em algumas situações (e.g., tirar fotos, medir ângulos de fraturas). Foi também observado a ausência de conexão $3 \mathrm{G}$ na maior parte do percurso visitado.

Apesar dos problemas encontrados, os resultados desse teste foram motivadores, pois mostraram que, com alguns ajustes e integração dos dados, os DMs podem ser utilizados no auxílio da realização de tarefas das aulas de campo. Por exemplo, a precisão dos dados coletados (coordenadas e ângulos de fraturas) é semelhante a das ferramentas usadas comumente (GPS e bússola) que custam muito mais caro do que o próprio DM. Alguns dos alunos, além do monitor, que experimentaram o dispositivo, demonstraram interesse em utilizá-los durante as aulas de campo tanto para acesso a informação complementar das aulas como para coleta de dados.

\section{Geomóvel}

\subsection{Princípios Norteadores}

A partir do experimento realizado, optou-se pela criação de um aplicativo móvel, o Geomóvel, que integrasse funcionalidades multimídia e de sensoriamento para apoiar os alunos do curso de graduação em Geologia durante as aulas de campo. As atividades escolhidas para integrar o aplicativo foram: as medições da rota e ângulo do mergulho, coleta de coordenadas geográficas, fotografias e anotações (via texto e/ou áudio).

Como mencionado anteriormente, na maioria dessas aulas, são realizadas viagens durante as quais os alunos realizam práticas de campo em diferentes pontos de estudo, tendo de realizar anotações em todos os momentos. Após essas viagens, os alunos precisam apresentar um relatório, que consolida as informações coletadas e os conhecimentos apreendidos. O Geomóvel auxilia os estudantes nessa tarefa final através da integração das informações anotadas e da exportação dos dados coletados. Além da integração das atividades em um mesmo aplicativo e da exportação dos dados coletados, dois requisitos não-funcionais nortearam o desenvolvimento do GeoMóvel: a necessidade de uso de interfaces multimodais e a exigência de sincronização oportuna das informações, devido a ausência de conexão internet na maior parte do trajeto. 


\subsection{Recursos e funcionalidades do Geomóvel}

O Geomóvel consiste em um aplicativo móvel compatível com dispositivos Android a partir da versão 2.3. Assim, permite-se que os alunos executem o aplicativo nas aulas de campo utilizando equipamentos com características variadas, seja em termos de tamanho, poder de processamento, memória ou recursos multimídia. O Geomóvel oferece várias modalidades de anotações baseada em áudio, texto e fotos. Por exemplo, através do teclado virtual do sistema Android, os alunos podem digitar anotações sobre os pontos de interesse do campo visitado. As informações também podem ser registradas via gravação de áudio, através do microfone do dispositivo móvel. Este recurso minimiza a dificuldade de registro textual e possibilita que os alunos gravem suas anotações sobre o fenômeno estudado. O aplicativo, quando requisitado, também invoca a câmera digital do dispositivo, proporcionando registros fotográficos dos afloramentos. Todas essas anotações são registradas em uma base de dados local do aplicativo e associadas a coordenadas geográficas.

O Geomóvel acessa também três tipos de sensores do dispositivo comuns a smartphones e tablets: o acelerômetro, o magnetômetro e o GPS. O Geomóvel utiliza o GPS contido no dispositivo para obter e gravar a localização (longitude e latitude) de onde as informações e medições estão sendo registradas As atividades de medição de ângulo e de rumo de mergulho usam o acelerômetro combinado ao magnetômetro para simular uma bússola. A Figura 2 apresenta algumas telas do aplicativo como a que simula uma bússola para medição da atitude e rumo de mergulho. A fim de evitar os problemas de visualização das medidas da bússola apontados no experimento exploratório, as interfaces dessas atividades foram concebidas de forma multimodal usando o sintetizador configurado como padrão no Android (e.g., eSpeak, SVOX, etc.). No momento da medição do rumo e do ângulo do mergulho, o Geomóvel pronuncia em português os valores que estão sendo capturados pelos sensores.
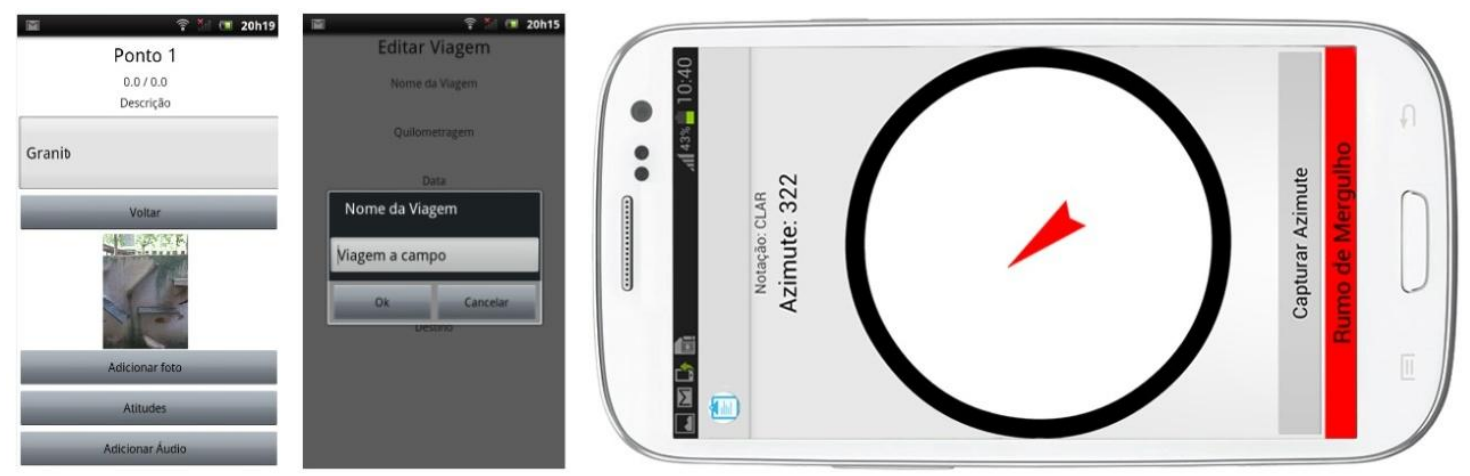

Figura 2- Algumas telas do aplicativo Geomóvel.

A Figura 3 exibe uma visão geral da utilização do aplicativo. Durante a aula de campo, o aluno utiliza o Geomóvel para coletar informações da viagem como um todo e dos pontos específicos a serem estudados. Primeiro, ele preenche os dados sobre a viagem, como a quilometragem do veículo, uma descrição e o ponto de destino. Ao longo da aula de campo, o aluno anota as informações de cada ponto a ser estudado. Podem ser registrados quantos pontos forem necessários. Para cada um, os dados a serem coletados são: descrição geral (que pode ser via texto e/ou via áudio), localização (através do GPS), rumo e ângulo do mergulho. Além disso, o aluno pode retirar fotos, associando-as com o ponto estudado, e compartilhá-las com os colegas via Bluetooth. 
Uma vez que uma conexão de dados está disponível, os alunos podem utilizar o aplicativo para gerar e exportar uma trajetória com as anotações e medições realizadas. Geomóvel gera os dados no formato padrão KMZ [Abdulrahman, 2008], que pode ser lido através de ferramentas gratuitas, tais como, Google Earth, GPS Map Viewer e FileViewPro. Após a geração do arquivo $\mathrm{KMZ}$, com todas as informações capturadas em campo, o Geomóvel permite que o aluno transmita o arquivo para um email préconfigurado. Essa transmissão dos dados pode ser feita no momento em que o aluno obter uma conexão com a Internet, em campo ou ao retornar da aula.

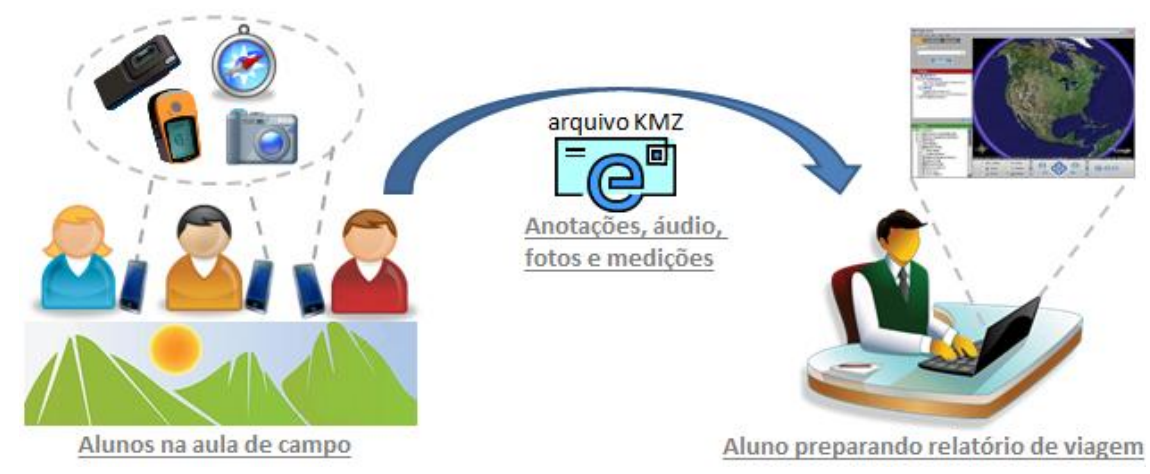

Figura 3- Visão geral da utilização dos recursos do Geomóvel.

\section{Estudo de Caso}

\subsection{Descrição}

Para avaliar este trabalho, foi realizado um estudo de caso do tipo exploratório [Yin, 2003]. O objetivo deste estudo foi obter indícios sobre a utilidade e os benefícios proporcionados pelo aplicativo Geomóvel para aulas de campo de geologia.

A amostra foi composta por três alunos e um professor do curso de Geologia. Este professor selecionado participou desde as fases de levantamento dos requisitos do aplicativo Geomóvel. Todos os alunos escolhidos eram do terceiro semestre do curso, ou seja, já haviam cursado mais de uma disciplina com aula de campo. Este estudo foi realizado dentro do campus da própria universidade em um local onde os professores de Geologia costumam levar os alunos para aulas práticas. Para o teste foram utilizados três smartphones, um com tela de 4" e dois com tela de 4,8", e um tablet com tela de $7 " 2$. As Figuras $4 \mathrm{a}$ e $4 \mathrm{~b}$ apresentam os alunos utilizando o aplicativo nesses dispositivos.

Antes de realizar o teste, o avaliador explicou os objetivos do estudo e a proposta de utilização do Geomóvel. Posteriormente, foi demonstrada a utilização do aplicativo com o preenchimento das informações e a utilização de todos os seus recursos. Após esta apresentação, o avaliador explicou como seria o teste: cada participante deveria cadastrar as informações da viagem, cadastrar a descrição do ponto de estudo, retirar fotos do ponto, gravar um áudio sobre o ponto e realizar as medições de rumo e ângulo do mergulho. Estas tarefas deveriam ser executadas duas vezes por cada participante. Depois da realização dos testes pelos participantes, cada um respondeu um questionário, onde deveria expressar a opinião sobre a satisfação com o aplicativo e a experiência com o seu uso. Após coletar os questionários e os dispositivos

\footnotetext{
${ }^{2}$ Os dispositivos móveis utilizados no teste foram: dois smartphones Samsung Galaxy SIII, um smartphone SonyErisson Xperia Acro IS11S e um tablet ASUS Google Nexus 7.
} 
móveis utilizados, o avaliador escolheu aleatoriamente um dos smartphones, acessou o Geomóvel e transmitiu o arquivo $\mathrm{KMZ}$ com as informações do teste. A Figura 4c mostra uma tela da ferramenta Google Earth após abertura do arquivo, observa-se a localização do Ponto no campus da [omitido para revisão cega], as informações digitadas no campo descrição do ponto e uma foto retirada no local do teste.

\subsection{Análise dos resultados}

Após a análise dos questionários e das anotações feitas durante o teste, pode-se concluir que os resultados do estudo de caso são animadores. Primeiramente, todos os participantes executaram as tarefas que foram pedidas com relação ao uso do Geomóvel. Sobre a utilização do dispositivo móvel e do aplicativo, os quatro participantes demonstraram boa receptividade e interesse em utilizá-los nas aulas de campo reais.

Os principais benefícios apontados pelos alunos foram: praticidade e facilidade de uso. Os depoimentos também indicam que eles concordam que o Geomóvel agiliza o registro dos dados durante as aulas de campo. Um dos alunos informou que já havia utilizado outros aplicativos de Geologia para Android, mas que o Geomóvel é mais indicado para as aulas de campo porque integra todas as informações importantes e tem exatamente o que é necessário. Outro aluno também informou que, às vezes, o departamento não tem bússolas ou equipamentos GPS disponíveis para as aulas, e com o aplicativo esse problema pode ser solucionado já que o custo de um smartphone Android é bem menor. $O$ professor de Geologia também ressaltou o aspecto da praticidade de integrar várias ferramentas (bússola, caderneta de anotação, câmera digital e GPS) em um único dispositivo. Outro aspecto importante destacado por ele foi a questão da objetividade do Geomóvel, cuja sistemática de uso conduz o aluno ao preenchimento correto das informações necessárias às aulas de campo.

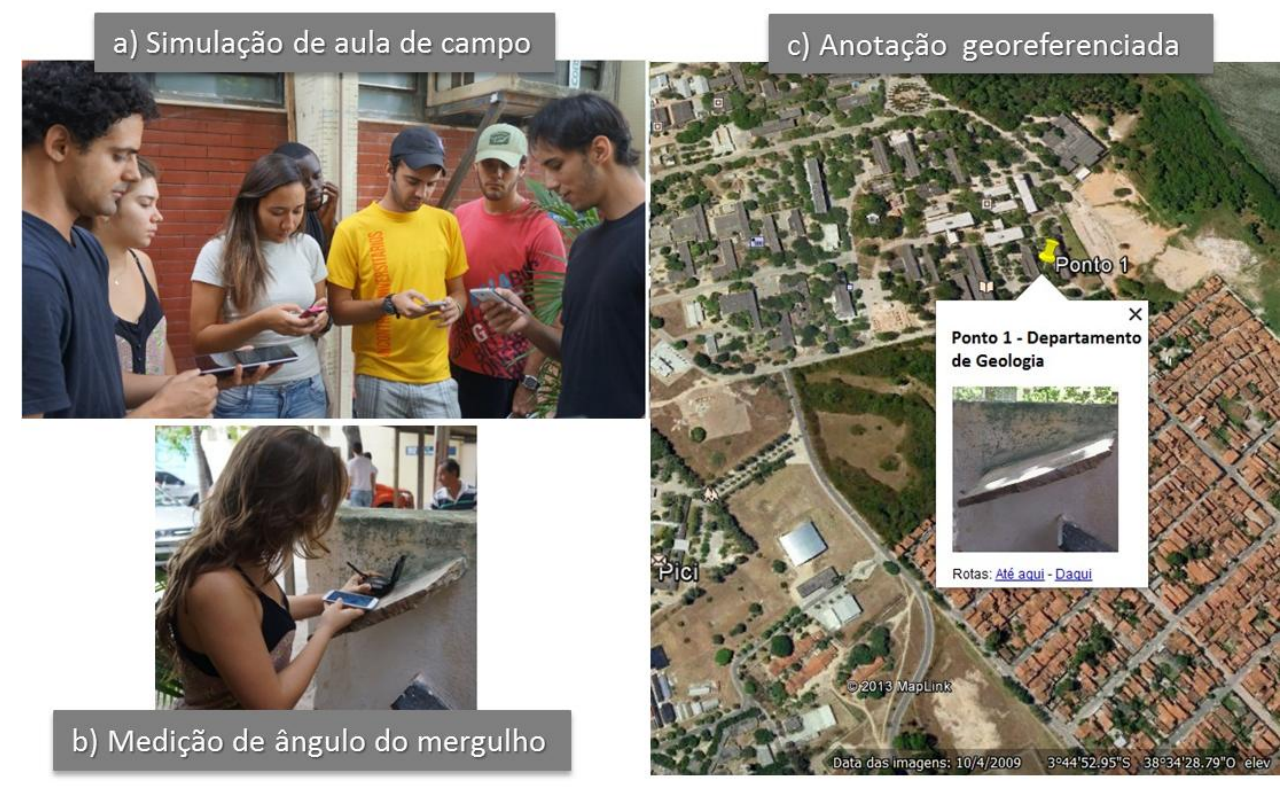

Figura 4 - A e B) Participantes utilizando o Geomóvel. C)Tela do Google Earth com visualização de informações coletadas no teste.

A Tabela 1 lista os dados de rumo e ângulo do mergulho obtidos pelo Geomóvel. Cada participante realizou duas vezes a medição do rumo/ângulo do mergulho do 
mesmo ponto. Durante o teste, o professor de Geologia utilizou sua bússola para realizar as mesmas medidas para servirem de parâmetro de comparação com os dados obtidos pelo aplicativo. Os valores obtidos com a bússola foram: $346 / 29$. Os valores do rumo do ângulo do mergulho são medidos em graus e variam de 0 a 360. Com relação à medida de ângulo de mergulho, observa-se que em quase todos os casos os valores obtidos com o Geomóvel são os mesmos que o obtido com a bússola (29 graus). Porém, no caso do rumo do mergulho, em algumas medições há uma diferença perceptível (-16 a + 27 graus). Consultado sobre as diferenças obtidas em alguns casos na medida de rumo do mergulho, o professor participante informou que os valores não são tão díspares e que talvez tenham ocorrido pelo fato do aplicativo Geomóvel não conter ainda uma bolha de nível que auxilia os alunos a realizar medições de rumo do mergulho mais precisas.

Tabela 1: Dados de rumo/ângulo do mergulho na notação Clar $^{3}$ obtidos no teste.

\begin{tabular}{|l|l|l|l|l|}
\hline & Aluno 1 & Aluno 2 & Aluno 3 & Professor \\
\hline $\mathbf{1}^{\text {a }}$ medição & $350 / 30$ & $360 / 29$ & $10 / 28$ & $13 / 29$ \\
\hline $\mathbf{2}^{\text {a }}$ medição & $330 / 29$ & $352 / 29$ & $348 / 29$ & $360 / 30$ \\
\hline
\end{tabular}

\section{Trabalhos Relacionados}

$\mathrm{Na}$ área de Geologia, algumas iniciativas têm sido desenvolvidas no sentido de promover a utilização dos dispositivos móveis. O aplicativo GeoMallorca é um guia para celulares, com textos e fotos, que permite que o usuário conheça sítios geológicos da ilha de Mallorca [Geo Mallorca, 2011]. Em Weng et al. (2012), os autores desenvolveram uma ferramenta para auxiliar os trabalhos em campo de um geólogo, permitindo a anotação das informações e a coleta de fotos e a localização via GPS. O software iGeology (2011) apresenta um mapa geológico da Grã-Bretanha. Com ele é possível obter informações como os fundamentos rochosos, as fendas e os depósitos de minerais superficiais dos solos britânicos. Ele também conta com imagens 3D para facilitar a compreensão da composição dos terrenos. Esses aplicativos se caracterizam como de uso geral para Geologia, diferentemente do Geomóvel que foi desenvolvido com foco nos aspectos específicos das aulas de campo.

Um estudo com aplicação das tecnologias móveis para o ensino de geologia com resultados exitosos é apresentado em Davies et al. (2010). Nesta pesquisa, os alunos são divididos em dois grupos: um que ficou no laboratório e outro que foi a campo com telefones celulares. Os participantes do segundo grupo faziam as anotações, tiravam fotos, gravavam vídeos e transmitiam para os alunos do primeiro grupo em tempo real. De um lado, os alunos captavam os dados que eram solicitados pelos alunos que estavam no laboratório, e do outro lado, os alunos do laboratório forneciam informações que eram importantes para auxiliar a aprendizagem dos que estavam em campo. As dificuldades encontradas nesse trabalho foram em coordenar as atividades cooperativas entre os dois grupos e a velocidade e a estabilidade da rede de dados do telefone celular. Infelizmente, esse cenário ainda é difícil de ser realizado na maioria das regiões de interesse geológico do interior do Brasil devido à ausência de conexão sem fio. Esse é

\footnotetext{
${ }^{3}$ A Notação Clar (ou Breithaupt) expressa a orientação tridimensional de uma feição geológica baseada na inclinação de um plano ou linha a ela pertencente.
} 
um dos motivos que levou ao Geomóvel ser desenvolvido para funcionar de forma offline na fase de coleta de dados.

\section{Considerações Finais e Trabalhos Futuros}

Iniciativas têm demonstrado os benefícios do emprego das tecnologias de computação móvel e ubíqua em diferentes áreas, como nas geociências. Este trabalho apresentou uma ferramenta para área de Geologia com foco nas aulas de campo e um estudo de caso para validá-la. $\mathrm{O}$ aplicativo desenvolvido, Geomóvel, mostrou-se como uma alternativa para contornar e minimizar as dificuldades que os alunos de Geologia enfrentam ao ter que utilizar diferentes ferramentas para realizar suas anotações durante as aulas de campo. De forma integrada e sistematizada, o Geomóvel permite que os alunos registrem as informações do campo, através de texto, áudio e fotos, realizem medições e obtenham a localização geográfica automaticamente. O Geomóvel também se apresenta como uma opção interessante para as aulas práticas ao se considerar que em grande parte dos departamentos de Geologia os equipamentos de GPS e bússola têm quantidade bem limitada e uso restrito. Os resultados do estudo de caso demonstraram os benefícios que o Geomóvel pode proporcionar nas aulas de campo. A interface amigável do aplicativo possibilitou que todos os participantes do teste concluíssem as tarefas que foram solicitadas. Tanto os alunos como o professor demonstraram satisfação em utilizar o aplicativo e mostraram-se motivados a usá-lo nas aulas de campo. Com relação ao comparativo dos valores obtidos dos sensores, observou-se que o Geomóvel apresentou resultados praticamente iguais quando comparando o ângulo do mergulho. Porém, os valores de rumo do mergulho apresentaram, em média, uma pequena diferença de 10 graus (para mais ou para menos).

Como trabalho futuro, pretende-se implementar no Geomóvel o recurso da bolha, já presente nas bússolas tradicionais. Esta é uma das possibilidades, apontada pelo professor participante do teste, para obter um valor mais preciso para o rumo de mergulho. Posteriormente, pretende-se realizar mais testes das funcionalidades do aplicativo para então realizar um novo estudo de caso em uma aula de campo real.

Por fim, outra possibilidade de pesquisa é aplicar o Geomóvel em outros cursos e analisar e comparar os resultados. Engenharia de Minas é um dos cursos que se planeja empregar o aplicativo sem modificações. Outros cursos que têm as aulas de campo como atividade pedagógica nos quais o Geomóvel também pode ser aplicado, com algumas alterações, são: Geografia, Biologia e Agronomia. Considerando a variedade de versões que podem vir a ser necessárias para cada curso, surge outra possibilidade de trabalho futuro: criar uma metodologia que possibilite que o próprio especialista/professor em uma determinada área do conhecimento possa criar e configurar sua aplicação móvel para ser utilizada nas aulas de campo. 


\section{Agradecimentos}

À Fundação Cearense de Apoio ao Desenvolvimento Científico e Tecnológico (FUNCAP) pelo financiamento ao Projeto "Sistemas Ubíquos e Sensíveis ao Contexto para o auxílio à aprendizagem em aulas de campo em Geociências" (projeto número PJP-0072-00091.01.00/12) e à Universidade Federal do Ceará (UFC) pelo financiamento ao Projeto "Uso da Tecnologia da Informação no Ensino de Geologia em Campo".

\section{Referências}

Abdulrahman, F.H. (2008) "Using KML files as encoding standard to explore locations, access and display data in Google Earth". Technical Paper. The Ohio State University, USA. Disponível em http://shoreline.eng.ohiostate.edu/publications/abdulrahman thesis.pdf.

Davies, S., Collins, T., Gaved M., Bartlett J., Valentine C. and McCann L. (2010) "Enabling Remote Activity: Using mobile technology for remote participation in geoscience fieldwork". In: Geophysical Research Abstracts, Vol. 12, 2010.

Geo Mallorca (2011) Disponível em http://pt.appbrain.com/app/geomallorca/com.letitguide.geomallorca.

iGeology (2011) "iGeology App | Britain's rocks in your pocket". Disponível em http://www.bgs.ac.uk/igeology/.

Marçal, E., Lima, L., Melo, A., Viana, W., Andrade, R. M. C.; Ribeiro J.W. (2010) “Da Elicitação de Requisitos ao Desenvolvimento de Aplicações de Mobile Learning em Matemática”, In: Simpósio Brasileiro de Informática na Educação, 2010.

Millard, D., Howard, Y., Gilbert, L. and Wills, G. (2009) "Co-design and Codeployment Methodologies for Innovative m-Learning Systems", In: Multiplatform E-Learning Systems and Technologies: Mobile Devices for Ubiquitous ICT-Based Education, IGI Global.

Sánchez J., Sáenz M., Muñoz, M., Ramirez G. e Martín S. (2009) Solite Situación actual del m-learning. Primer Informe Público. Disponível em: http://remo.det.uvigo.es/solite/

Sharples, M. (2007). "Big issues in mobile learning. learning sciences research institute”. Disponível em http://matchsz.inf.elte.hu/tt/docs/Sharples-20062.pdf.

Teixeira, W., Toledo, C. M., Fairchild, T.R. e Taioli, F. (2000) "Decifrando a Terra". Oficina de Textos, São Paulo, p. 558.

Viana, W., Andrade, R. M. C., Maia, M., Marçal, E., Gensel, J., Lima, J. V, Junior, J. C. e Sanchez, J. (2011) . Towards context-aware and mobile e-learning application. In: TISE 2011, Congreso Internacional de Informática Educativa, 2011, Santiago, Chile.

Weng, Y.H., Sun, F.S., Grigsb J.D. (2012). "GeoTools: An android phone application in geology". In: Computers \& Geosciences, Volume 44, July 2012, Pages 24-30.

Yin, R. K. (2003) "Case study research: design and methods" 3rd ed. Thousand Oaks : Sage Publications, 2003. 